\title{
Distribuição altitudinal e sazonal de afídeos (Hemiptera, Aphididae) na Serra do Mar, Paraná, Brasil ${ }^{1}$
}

\author{
Sonia M. N. Lazzari $^{2} \&$ Crisleide M. Lazzarotto ${ }^{2}$ \\ ${ }^{1}$ Contribuição número 1523 do Departamento de Zoologia, Universidade Federal do Paraná. \\ 2 Departamento de Zoologia, Universidade Federal do Paraná. Caixa Postal 19020, 81531-980 Curitiba, Paraná, Brasil. \\ E-mail: lazzari@ufpr.br; cmlazza@ig.com.br
}

\begin{abstract}
There are about 130 species of aphids registered for Brazil and most of them are exotic, originated from temperate regions. The objective of this research was to survey the aphid species that occur in different altitudes in the rich and diverse ecosystem of the Atlantic Rain Forest. The area surveyed included 11 collecting points ranging from -10 to $1000 \mathrm{~m}$ a.s.l. in Serra do Mar, in Paraná State, using yellow pan traps and plant collection, during one year. A total of 87 aphid species were collected in the traps and plants. The richness of species was noticeable high in transitional areas, where different plant communities overlapped. The most abundant aphid species was Aphis spiraecola Patch, 1914, in every collecting place. Population peaks occurred mainly in September when most plants showed new shoots. It was not observed an altitudinal gradient pattern of aphid distribution in the Serra do Mar, but some species were found to be restricted to specific altitudes. It is known that climate, besides other biotic and abiotic factors, affect aphid population fluctuation, but no correlation was established with temperature and rain fall.
\end{abstract}

KEY WORDS. Aphid fauna, Atlantic Rain Forest, insect survey, spatial distribution, yellow pan traps.

\begin{abstract}
RESUMO. Distribuição altitudinal e sazonal de afídeos (Hemiptera, Aphididae) na Serra do Mar, Paraná, Brasil. Dentre as 130 espécies de afídeos registradas para o Brasil, a maioria é exótica com origem em regiões temperadas. O objetivo desta pesquisa foi o estudo das espécies de afídeos que ocorrem em diferentes altitudes no ecossistema rico em diversidade da Mata Atlântica. Onze pontos de coleta foram amostrados em um gradiente altitudinal de -10 a 1000 m s.n.m. na Serra do Mar, no estado do Paraná com armadilhas amarelas de água e coletas nas plantas no decorrer de um ano. Um total de 87 espécies de afídeos foi coletado em armadilhas e em plantas. O maior número de espécies foi encontrado em áreas de transição ou ecótone definidos pela sobreposição de comunidades diferentes de plantas. Aphis spiraecola Patch, 1914 foi a espécie mais abundante registrada em todos os locais amostrados. O pico populacional ocorreu, principalmente, no mês de setembro quando as plantas estavam em brotação. Não foi observado um padrão altitudinal na distribuição dos afídeos na Serra do Mar, porém algumas espécies foram registradas em altitudes específicas. Condições climáticas, além de outros fatores bióticos e abióticos, afetam a flutuação populacional dos afídeos, porém não foi possível estabelecer uma correlação entre a ocorrência dos insetos com a temperatura e precipitação.
\end{abstract}

PALAVRAS CHAVE. Afidofauna, armadilhas amarelas de água, distribuição espacial, Floresta Atlântica.

Mesmo sendo uma região de alto valor científico e social por ter características de uma floresta tropical, ainda são muito escassos os trabalhos na Mata Atlântica, particularmente na Serra do Mar, tanto com relação aos aspectos mais gerais da fauna, quanto ao estudo de grupos mais específicos, como é o caso dos afídeos. Para este grupo de insetos exclusivamente fitófagos há apenas uma referência sobre sua riqueza e diversidade na Serra do Mar, Paraná (LAzzArotTo \& LAzZARI 1998).

Alguns pesquisadores têm concentrado a atenção na discussão da alta riqueza de espécies de afídeos no Hemisfério
Norte, contrastada com a baixa riqueza nas latitudes do Hemisfério Sul. No Brasil são registradas apenas 3,3\% das espécies catalogadas mundialmente (COSTA et al. 1993 e SousA-Silva \& ILHARCo 1995). Dixon et al. (1987) discutem que a pequena quantidade de afídeos nos trópicos é devida à grande quantidade de espécies de plantas, tendo os afídeos baixa eficiência em localizar seu hospedeiro. De acordo com a quantidade de plantas hospedeiras, Dixon \& Kindlmann (1990) sustentam a idéia de que a abundância das plantas é o principal fator que determina a abundância dos afídeos. Heıe (1994) relata que os Aphididae 
são um grupo pouco especializado para explorar novos ambientes e MaCKenzie et al. (1994) afirmam que nas latitudes do sul há um declínio no número de espécies de afídeos.

No Brasil, Moreira (1925) e Costa Lima (1942) catalogaram as espécies mais comuns de afídeos e suas plantas hospedeiras, incluindo em seus estudos alguns aspectos biológicos. SiLVA et al. (1968) também citam diversas espécies de afídeos e seus inimigos naturais. CostA et al. (1993) apresentaram uma lista com chave de identificação para 115 espécies de afídeos (com aproximadamente 3937 espécies). Mais recentemente, SousA-SiLVA \& ILHARCO (1995) elaboraram uma lista preliminar com 116 espécies de afídeos do Brasil e suas plantas hospedeiras.

A flutuação sazonal dos afídeos está relacionada com o hábito alimentar, disponibilidade e fenologia das plantas hospedeiras e também com fatores climáticos. As espécies polífagas podem distribuir-se mais amplamente, tanto temporal quanto espacialmente. De acordo com Cermeli (1970), algumas espécies podem estar presentes durante todo o ano, mas com épocas de maior incidência, enquanto outras ocorrem em apenas uma estação do ano. Segundo WoldA (1978), a sazonalidade e abundância de grupos de insetos podem ser estritamente controladas pela disponibilidade de alimento, sendo que qualquer distúrbio no ambiente pode afetar a quantidade e qualidade de alimento disponível e, conseqüentemente, a flutuação das espécies.

Os fatores climáticos aos quais os afídeos estão expostos no ambiente afetam sensivelmente suas populações, incrementando-as ou suprimindo-as. FAGUNDES \& ARNT (1976) obtiveram, para Macrosiphum avenae (Fabricius, 1775), maior capacidade reprodutiva quando criados a uma temperatura de $20^{\circ} \mathrm{C}$, enquanto que os pulgões criados a uma temperatura de $30^{\circ} \mathrm{C}$ não concluíram a fase ninfal. RoBERT (1987) relata que cada espécie de afídeo possui seus limites inferiores e superiores de temperatura para que possam voar.

O objetivo desta pesquisa foi verificar se há padrões de distribuição altitudinal e sazonal de afídeos na Serra do Mar, influenciados por características vegetacionais e climáticas e, desta forma, contribuir para o conhecimento da fauna deste ecossistema tão particular.

\section{MATERIAL E MÉTODOS}

As coletas de afídeos foram realizadas com armadilhas amarelas de água, tipo Möericke, quinzenalmente, no período de dezembro de 1993 a dezembro de 1994. As armadilhas foram confeccionadas com bacias plásticas de $34 \mathrm{~cm}$ de diâmetro e $11 \mathrm{~cm}$ de profundidade de cor amarela e a borda pintada de marrom, com orifícios vedados com tela de nylon, para evitar o transbordamento e perda dos insetos em caso de chuvas. As armadilhas eram colocadas em suporte de madeira a 1,2 $\mathrm{m}$ do solo. Cada armadilha era preenchida com 2,5 L de água, adicionando-se algumas gotas de detergente para quebrar a tensão superficial e $10 \mathrm{~mL}$ de formol a $10 \%$, a fim de conservar os insetos por mais tempo. Os afídeos eram retirados das armadilhas com um pincel fino e conservados em etanol a 70\%; pos- teriormente eram triados e montados em lâminas permanentes, seguindo a metodologia de MARTIN (1983), com pequenas modificações. O material foi identificado a nível específico e as lâminas depositadas na Coleção de Entomologia Pe. J.S. Moure, Departamento de Zoologia, Universidade Federal do Paraná, Curitiba, Paraná, Brasil (DZUP).

As espécies de plantas num raio de 100 a 150 m das armadilhas eram examinadas esporadicamente, e as folhas ou galhos com pulgões eram coletados e colocados em sacos plásticos com toalha de papel, anotando-se a data e local de coleta. No laboratório, os pulgões eram retirados das plantas e colocados em frascos com etanol a 70\%, para posterior preparação e identificação.

As coletas foram efetuadas em onze pontos da Mata Atlântica, no Paraná. Os locais de 1 a 5 localizavam-se na Estação do Instituto Agronômico do Paraná (IAPAR) em Morretes caracterizados pela floresta ombrófila densa atlântica de terras baixas - locais 1 (-10 m s.n.m.), 2 (10 m s.n.m.), 3 (20 m s.n.m.) e floresta ombrófila densa atlântica submontana - locais $4(65 \mathrm{~m}$ s.n.m.) e 5 (78 m s.n.m.). As armadilhas foram instaladas em áreas abertas nos locais 1 e 2 sendo influenciadas por vegetação secundária bastante avançada. Nos locais 3, 4 e 5, as armadilhas foram instaladas em meio a vegetação secundária em estágios iniciais e médio de desenvolvimento.

Os locais 6, 8, 9 e 10 localizavam-se na Estrada da Graciosa, respectivamente a 85, 190, 555 e 750 m s.n. Os três primeiros eram caracterizados por floresta ombrófila densa atlântica submontana com influência da vegetação secundária predominando as plantas herbáceas; enquanto o local 10 era caracterizado pela transição entre floresta ombrófila mista (floresta de araucária) e floresta ombrófila densa atlântica (floresta atlântica) em contato com uma vegetação de estágios sucessionais iniciais (espécies pioneiras).

Os locais 7 (185 m s.n.m.) e 11 (1000 m s.n.m.) localizavam-se na BR 277 (Rodovia Curitiba-Paranaguá) caracterizados pela floresta ombrófila densa atlântica montana em transição com a floresta ombrófila mista. O local 7 era influenciado pela ocorrência de espécies frutíferas cultivadas e por ampla área de pastagem e o local 11 circundado por áreas de floresta secundária.

Dados de temperatura e precipitação pluviométrica foram obtidos através da Estação Meteorológica do IAPAR, Morretes e da Estação Véu de Noiva, próximas das áreas de coleta.

\section{RESULTADOS E DISCUSSÃO}

Foram coletados 8019 espécimes alados de afídeos nas onze armadilhas amarelas de água, no período de 12 meses, distribuídas em altitudes distintas da Serra do Mar. A lista das espécies é apresentada em LazzarotTo \& Lazzari (1998).

Os locais 5, 7, 9, 1 e 6 foram, em ordem decrescente, os mais representativos em termos de total de indivíduos, representando, cada um, mais de $10 \%$ do total de afídeos coletados (Tab. I). Estes locais caracterizam-se por nítidas transições entre comunidades vegetais. Segundo Odum (1988), uma transição nítida entre duas ou mais comunidades vegetais é denomi- 
Tabela I. Número total de afídeos alados coletados com armadilhas amarelas de água em diferentes altitudes da Serra do Mar, PR, no período de dezembro de 1993 a dezembro de 1994 (Locais 01 a 09 e 11) e de abril de 1994 a dezembro de 1994 (Local 10).

\begin{tabular}{|c|c|c|c|c|c|c|c|c|c|c|c|c|c|c|c|c|}
\hline \multirow{2}{*}{$\begin{array}{c}\text { Locais } \\
\text { (m s.n.m.) }\end{array}$} & \multirow{2}{*}{$\begin{array}{c}1993 \\
\text { Dez }\end{array}$} & \multicolumn{12}{|c|}{1994} & \multirow{2}{*}{$\begin{array}{c}\text { Total } \\
\text { exemplares }\end{array}$} & \multirow{2}{*}{$\%$} & \multirow{2}{*}{$\begin{array}{l}\text { Total de } \\
\text { espécies }\end{array}$} \\
\hline & & Jan & Fev & Mar & Abr & Mai & Jun & Jul & Ago & Set & Out & Nov & Dez & & & \\
\hline $01(-10)$ & 225 & 274 & 19 & 39 & 51 & 32 & 25 & 8 & 18 & 192 & 33 & 36 & 22 & 974 & 12,1 & 31 \\
\hline $02(10)$ & 128 & 56 & 17 & 126 & 129 & 48 & 29 & 10 & 38 & 97 & 27 & 26 & 29 & 760 & 9,5 & 34 \\
\hline $03(20)$ & 32 & 59 & 5 & 26 & 58 & 17 & 12 & 6 & 18 & 127 & 23 & 13 & 8 & 404 & 5,0 & 27 \\
\hline $04(65)$ & 26 & 28 & 12 & 11 & 17 & 5 & 7 & 4 & 7 & 130 & 17 & 12 & 13 & 289 & 3,6 & 27 \\
\hline 05 (78) & 58 & 97 & 18 & 55 & 93 & 78 & 55 & 59 & 101 & 680 & 106 & 79 & 59 & 1538 & 19,2 & 41 \\
\hline $06(85)$ & 50 & 81 & 10 & 11 & 44 & 25 & 14 & 24 & 45 & 370 & 50 & 50 & 61 & 835 & 10,4 & 47 \\
\hline $07(185)$ & 131 & 238 & 20 & 159 & 58 & 36 & 30 & 10 & 16 & 235 & 59 & 40 & 34 & 1066 & 13,3 & 35 \\
\hline 08 (190) & 33 & 215 & 12 & 18 & 47 & 38 & 21 & 8 & 16 & 56 & 28 & 19 & 16 & 527 & 6,6 & 23 \\
\hline $09(555)$ & 33 & 68 & 14 & 35 & 28 & 17 & 14 & 46 & 177 & 483 & 24 & 65 & 14 & 1018 & 12,7 & 36 \\
\hline $10(750)$ & - & - & - & - & 111 & 45 & 12 & 4 & 2 & 20 & 16 & 17 & 29 & 256 & 3,2 & 18 \\
\hline $11(1000)$ & 12 & 08 & 11 & 16 & 31 & 20 & 3 & 5 & 18 & 199 & 18 & 8 & 3 & 352 & 4,4 & 41 \\
\hline Total & 728 & 1124 & 138 & 496 & 667 & 361 & 222 & 184 & 456 & 2589 & 401 & 365 & 288 & 8019 & 100 & 87 \\
\hline
\end{tabular}

nada de ecótone e, freqüentemente, há a tendência para uma maior riqueza e densidade populacional de algumas destas espécies; sendo essa tendência conhecida como efeito de borda. A armadilha do local 5, a 78 m s.n.m., onde se registrou o maior número de espécimes de afídeos, estava instalada em um local aberto, facilitando a atração dos afídeos pela armadilha amarela colocada sobre um fundo escuro. LieweHr \& CRANSHAW (1991), comparando armadilhas instaladas em diferentes padrões de solo, encontraram maior quantidade de afídeos em solo nu ou com um fundo escuro, como era o caso desse local.

Os locais menos representativos, com menos de $5 \%$ do total de afídeos coletados, foram 4, 10 e 11 . O local 4, a $65 \mathrm{~m}$ s.n.m., caracteriza-se por ser praticamente um corredor de vento que pode diminuir a freqüência do vôo dos afídeos, pois, segundo Walters \& Dixon (1984), a freqüência de vôo dos afídeos diminui com o aumento da velocidade do vento. No local 10, a 750 m s.n.m., além das coletas terem iniciado somente em abril, a área era roçada periodicamente, não tendo uma influência contínua das espécies vegetais invasoras, as quais poderiam abrigar diversas espécies de afídeos. O local 11 era o de altitude mais elevada (1000 m s.n.m.), com condições climáticas comparativamente mais rigorosas e com cobertura vegetal mais restrita, condições essas que, segundo НЕвеRт (1980), afetam diretamente o tamanho das populações.

Quanto à riqueza de espécies, os locais 6, 5 e 11 apresentaram, em ordem decrescente, mais espécies de afídeos (Tab. I), correspondendo, respectivamente, às altitudes de 85, 78 e 1000 m s.n.m. Estudos de LAZZAROTTO \& LÁZZARI (1998), registraram maior diversidade e equitabilidade de afídeos nessas mesmas áreas, mas uma maior homogeneidade na distribuição dos indivíduos nas espécies e menor índice de diversidade de afídeos no local 10 a 750 m s.n.m., com a segunda menor equitabilidade. Assim, não foi possível estabelecer um padrão no gradiente altitudinal dos afídeos na área estudada, pois outros fatores, não avaliados nesta pesquisa, podem influenciar na distribuição dos afídeos.

Turner (1974), estudando a diversidade e distribuição de populações de insetos da Ordem Psocoptera em diferentes altitudes na Jamaica, entre 152 m e 1220 m s.n.m., obteve maior número de espécies com o aumento da altitude; assim como a riqueza e abundância de um gênero de Coleoptera foram maiores a uma altitude de 1500 m (Ribeiro et al. 1994). Porém, segundo outros autores (Begon et al. 1990 e Fernandes \& Price 1991), a riqueza de espécies declina, de um modo geral, com o aumento da altitude. Em trabalhos com grupos específicos de insetos, como os de Hebert (1980) e Randall (1982), com lepidópteros, e FERNANDES \& LARA (1993), com insetos galhadores, foi observada maior diversidade de insetos em altitudes mais baixas. Wolda (1987) encontrou um decréscimo gradual da riqueza e abundância de insetos pertencentes a diversas ordens em um gradiente altitudinal entre 100-2200 m. LAWTON et al. (1987) não encontraram mudanças marcantes na abundância e riqueza de espécies de insetos herbívoros alimentando-se em um tipo de fanerógama ao longo de um gradiente altitudinal de 0 a 300 m s.n.m., apenas uma das espécies apresentou sua abundância reduzida com o aumento da altitude, a qual não possuía sincronismo com sua planta hospedeira, pois o desenvolvimento desta era retardado nas altitudes elevadas.

Algumas espécies ocorreram exclusivamente na altitude de 1000 m s.n.m.: Carolinaia caricis Wilson, 1911, Geoica lucifuga (Zehntner, 1897), Hyperomyzus carduellinus (Theobald, 1915), Therioaphis trifolii (Monnel, 1882) e Uroleucon gravicorne (Patch, 1919). Holman (1974) também registrou C. caricis e U. gravicorne somente nas regiões montanhosas de Cuba. Por outro lado, Sitobion salviae (Bartholomew, 1932) foi registrada somente no local de altitude próxima à do nível do mar, provavelmente relacionada com a presença de sua planta hospedeira. 

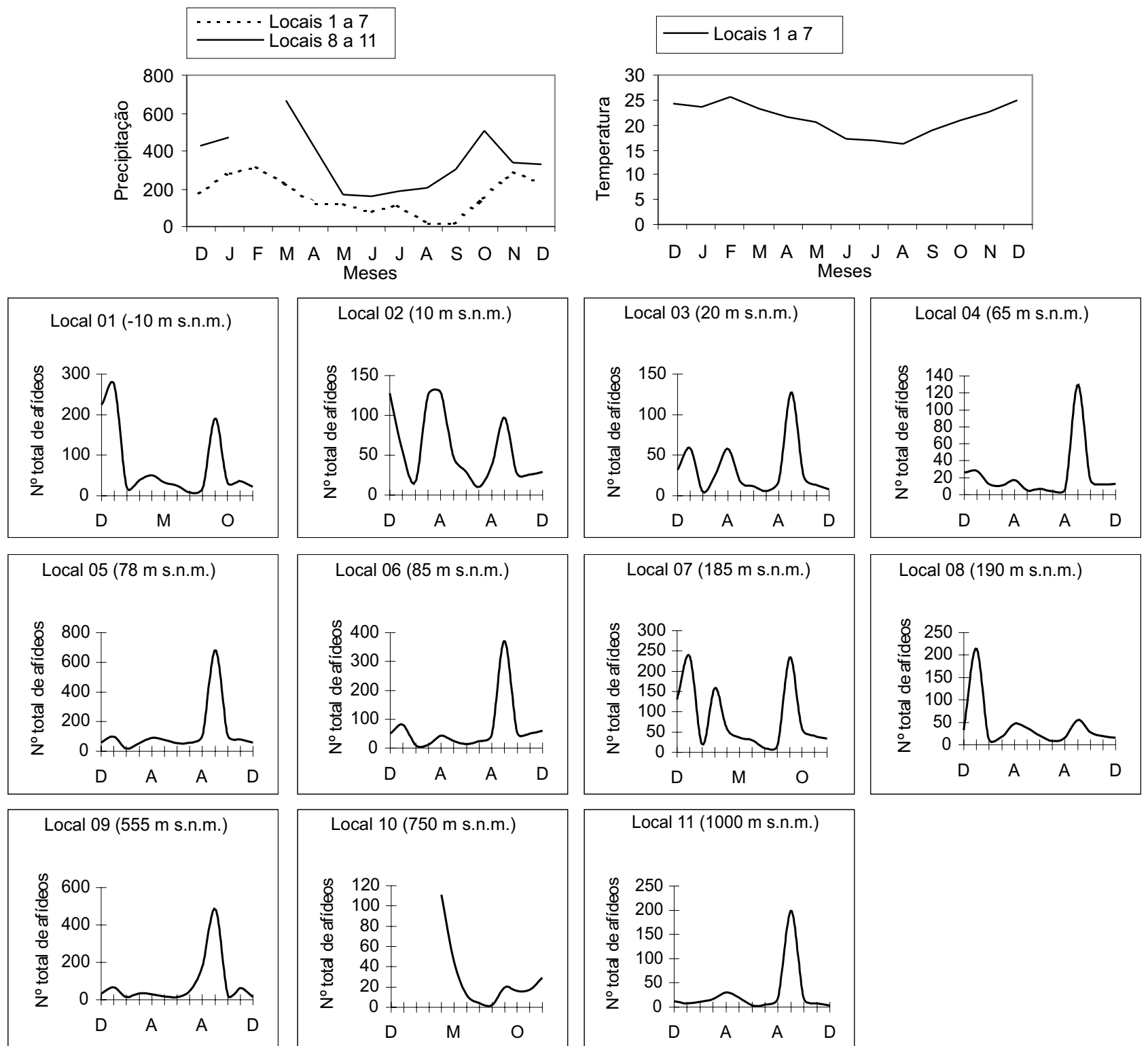

Figura 1. Flutuação sazonal de afídeos coletados com armadilhas amarelas de água em 11 locais da Serra do Mar, Paraná, no período de dezembro de 1993 a dezembro de 1994 (Locais 1 a 9 e 11) e de abril de 1994 a dezembro de 1994 (local 10) em um gradiente altitudinal de -10 a 1000 m s.n.m. com indicação de precipitação e temperatura obtidos da Estação Experimental do IAPAR, Morretes (locais 1 a 7) e da Estação Véu da Noiva (locais 8 a 11). Vide texto para caracterização dos locais de coleta.

O maior número de espécies de afídeos foi registrado no mês de setembro, na maioria dos locais (Fig. 1), em decorrência da fenologia das plantas em fase de brotação, com folhas mais tenras que propiciam a alimentação e reprodução dos afídeos; além de temperaturas amenas e baixa precipitação no ano das coletas. Apenas nos locais 8 e 10, a maior quantidade de espécies foi nos meses de janeiro e maio, respectivamente.

As espécies Coloradoa rufomaculata (Wilson, 1908),
Metopolophium dirhodum (Walker, 1849), Ovatus crataegarius (Walker, 1850), associadas a gramíneas, e T. trifolii, a leguminosas, foram coletadas somente no mês de setembro (Tab. II), sendo que destas, a primeira espécie foi registrada em cinco dos locais de coleta e as demais estiveram presentes em apenas um local.

Quanto ao número de indivíduos, o mês mais representativo também foi setembro, com temperatura média de $18,8^{\circ} \mathrm{C}$ 
Tabela II. Relação das espécies de afídeos coletadas com armadilhas amarelas de água, que tiveram registro apenas no mês de setembro, 1994, em locais da Serra do Mar, Paraná.

\begin{tabular}{|c|c|c|c|c|c|c|c|c|c|c|c|}
\hline \multirow{2}{*}{ Espécies } & \multicolumn{11}{|c|}{ Locais m s.n.m. } \\
\hline & -10 & 10 & 20 & 65 & 78 & 85 & 185 & 190 & 555 & 750 & 1000 \\
\hline \multicolumn{12}{|c|}{ Coloradoa rufomaculata } \\
\hline \multicolumn{12}{|c|}{ Metopolophium dirhodum } \\
\hline \multicolumn{12}{|l|}{ Ovatus crataegarius } \\
\hline Therioaphis trifolii & & & & & & & & & & & \\
\hline
\end{tabular}

e precipitação de $14,6 \mathrm{~mm}^{3}$, para os locais 3, 4, 5, 6 situados no município de Morretes, e valores médios da precipitação em $301,3 \mathrm{~mm}^{3}$ para os locais 9 e 11, situados na Estrada da Graciosa e no município de São José dos Pinhais (Fig. 1). Para os demais locais, 1, 2, 7, 8 e 10, os picos ocorreram nos meses de dezembro de 1993, janeiro, março e abril com temperaturas e precipitações mais elevadas (Fig. 1).

Um total de 19 espécies de afídeos foram coletadas em 17 famílias de plantas hospedeiras, nos diversos locais (Tab. III). Destas, Hysteroneura setariae e Uroleucon cordobense foram coletadas apenas nas plantas, não sendo amostradas com armadilhas.

A presença de $A$. spiraecola foi registrada em $50 \%$ das plantas amostradas, justificando sua presença como espécie comum nas armadilhas. Recentemente $A$. spiraecola foi registrada em $67 \%$ das plantas coletadas no México a uma altitude de $1600 \mathrm{~m}$ no período de 1996 a 2000 (Trejo-Loyo et al. 2004). Pentalonia nigronervosa, foi observada em todos os locais em que Hedichyum coronarium Koenig (lírio-do-brejo) estava presente, ou seja, em altitudes mais baixas com característica brejosa. A presença desta espécie nas armadilhas também foi registrada até a altitude de 550 m s.n.m. (local 9). A espécie Uroleucon muermosum só obteve registro no local 10 (750 m s.n.m.), tanto nas plantas quanto nas armadilhas.

Os gêneros da família Asteraceae como: Baccharis spp., Eupatorium spp. e Vernonia spp. foram os que apresentaram mais espécies de afídeos. De acordo com EAsTop (1978), as plantas hospedeiras preferenciais dos Aphidoidea monófagos pertencem à família Asteraceae.

\section{CONCLUSÕES}

Não se observou um padrão de distribuição altitudinal das espécies de afídeos na Serra do Mar, porém algumas espécies, destacando-se Aphis spiraecola, apresentaram uma ampla distribuição espacial e temporal, enquanto outras estavam restritas a determinadas altitudes;

O pico populacional das espécies de afídeos, na maioria dos locais, foi em setembro; influenciado pela fenologia das plantas que favorece o desenvolvimento das populações, pela temperatura amena e precipitação moderada. Fevereiro e março foram os meses com o menor número de indivíduos e de espécies, possivelmente devido à alta precipitação no período;
Tabela III. Lista das famílias de plantas hospedeiras coletadas com afídeos em trechos da Serra do Mar, Paraná, no período de setembro de 1993 a setembro de 1995.

\begin{tabular}{|c|c|}
\hline Família & Afídeos \\
\hline Aquifoliaceae & Toxoptera aurantii (Boyer de Fonscolombe, 1907) \\
\hline \multirow[t]{14}{*}{ Asteraceae } & Aphis coreopsidis (Thomas, 1878) \\
\hline & Aphis fabae Scopoli, 1763 \\
\hline & Aphis gossypii Glover, 1877 \\
\hline & Aphis spiraecola Patch, 1914 \\
\hline & Aulacorthum solani (Kaltenbach, 1843) \\
\hline & Brachycaudus helechrysi (Kaltenbach, 1843) \\
\hline & Tetraneura nigriabdominalis (Sasaki, 1899) \\
\hline & Toxoptera aurantii (Boyer de Fonscolombe, 1907) \\
\hline & Uroleucon ambrosiae (Thomas, 1878) \\
\hline & Uroleucon compositae (Theobald, 1915) \\
\hline & Uroleucon cordobense (E.E. Blanchard, 1932) \\
\hline & Uroleucon gravicorne (Patch, 1919) \\
\hline & Uroleucon muermosum (Essig, 1953) \\
\hline & Uroleucon sonchi (Linnaeus, 1767) \\
\hline Bignoniaceae & Aulacorthum solani (Kaltenbach, 1843) \\
\hline Cucurbitaceae & Aphis spiraecola Patch, 1914 \\
\hline Euphorbiaceae & Toxoptera aurantii (Boyer de Fonscolombe, 1907) \\
\hline \multirow[t]{2}{*}{ Leguminosae } & Aphis gossypii Glover, 1877 \\
\hline & Aphis spiraecola Patch, 1914 \\
\hline Loranthaceae & Toxoptera aurantii (Boyer de Fonscolombe, 1907) \\
\hline \multirow[t]{2}{*}{ Melastomataceae } & Aphis spiraecola Patch, 1914 \\
\hline & Toxoptera aurantii (Boyer de Fonscolombe, 1907) \\
\hline Musaceae & Pentalonia nigronervosa Coquerel, 1859 \\
\hline Myrtaceae & Aphis spiraecola Patch, 1914 \\
\hline Plantaginaceae & Aphis gossypii Glover, 1877 \\
\hline \multirow[t]{3}{*}{ Poaceae } & Histeroneura setariae (Thomas, 1878) \\
\hline & Rhopalosiphum maidis (Fitch, 1856) \\
\hline & Tetraneura nigriabdominalis (Sasaki, 1899) \\
\hline \multirow[t]{2}{*}{ Rosaceae } & Aphis spiraecola Patch, 1914 \\
\hline & Aulacorthum solani (Kaltenbach, 1843) \\
\hline Rubiaceae & Toxoptera citricidus (Kirkaldy, 1907) \\
\hline Saxifragaceae & Aphis spiraecola Patch, 1914 \\
\hline Solanaceae & Aphis solanella Theobald, 1914 \\
\hline Zingiberaceae & Pentalonia nigronervosa Coquerel, 1859 \\
\hline
\end{tabular}


Espécies da família Asteraceae, presentes em praticamente todos os locais próximos às armadilhas, apresentaram a maior riqueza de espécies de afídeos;

Em geral, pode-se afirmar que a distribuição e flutuação sazonal dos afídeos foram influenciadas pelas condições climáticas, presença ou ausência das plantas hospedeiras e seu estado fenológico, além de fatores bióticos e abióticos que não foram avaliados nesta pesquisa.

\section{AGRADECIMENTOS}

Ao Prof. Dr. Sandro Menezes da Silva, do Departamento de Botânica da UFPR, pela caracterização e descrição florística dos locais. Ao IAPAR em Morretes, à polícia rodoviária e florestal na Estrada da Graciosa e aos proprietários do Restaurante Bela Vista na BR277, por permitirem as coletas nas suas propriedades. Ao CNPq pela concessão de bolsa de mestrado para a segunda autora.

\section{REFERÊNCIAS BIBLIOGRÁFICAS}

Begon, M.; J.L. Harper \& C.R. Townsend. 1990. Ecology, individuals, populations and communities. Blackwell Scientific Publications. $2^{\text {nd }}$ ed., 945p.

Cermeli, M. 1970. Notas preliminares sobre la fluctuacion de áfidos en Cagua, Estado Aragua, Venezuela. Agronomie Tropicale, Paris, XX: 311-321.

Costa Lima, A.M. 1942. Insetos do Brasil. Homópteros. Rio de Janeiro, Escola Nacional de Agronomia, Imprensa Nacional, $327 \mathrm{p}$.

Costa, C.L.; V.F. Eastop \& R.L. Blackman. 1993. Brazilian Aphidoidea: I. Key to families, subfamilies and account of the Phylloxeridae. Pesquisa Agropecuária Brasileira, Brasília, 28: 197-215.

EASTOP, V.F. 1978. Diversity of the Sternorrhyncha within major climatic zones. Symposium of the Royal Entomological Society of London 9: 71-88.

Dixon, A.F.G. \& P. KindLmann. 1990. Role of plant abundance in determining the abundance of herbivorous insects. Oecologia, Danvers, 83: 281-283.

Dixon, A.F.G.; P. Kindlmann; J. Leps \& J. Holman. 1987. Why there are so few species of aphids, especially in the tropics. American Naturalist, Chicago, 129: 580-592.

Fagundes, A.C. \& T.A. Arnt. 1976. Efeitos de diferentes temperaturas sobre o pulgão da espiga Macrosiphum avenae (F.). Agronomia Sulriograndense, Porto Alegre, 12: 59-64.

Fernandes, G.W. \& A.C.F. Lara. 1993. Diversity of indonesian gall-forming herbivores along altitudinal gradients. Biodiversity Letters, Manchester, 1: 186-192.

Fernandes, G.W. \& P.W. Price. 1991. Comparison of tropical and temperate galling species richness: the roles of environmental harshness and plant nutrient status, p. 91-115. In: P.W. Price; T.M. Lewinsohn; G.W. Fernandes \& W.W. Benson (Eds). Plantanimal interactions. Evolutionary ecology in tropical and temperate regions. New York, John Wiley \& Sons, 713p.

Hebert, P.D.N. 1980. Moth communities in montane Papua New Guinea. Journal of Animal Ecology, Oxford, 49: 593-602.

HeIE. O.E. 1994. Why are there so few aphid species in the temperate areas of the southern hemisphere? European Journal Entomology, Ceske Budejovice, 91: 127-133.

Holman, J. 1974. Los áfidos de Cuba. La Habana, Instituto Cubano del Libro, 304p.

Lazzarotto, C.M. \& S.M.N. LazZari. 1998. Richness and diversity of aphids (Homoptera, Aphididae) along an altitudinal gradient in the Serra do Mar, Paraná, Brazil. Revista Brasileira de Zoologia, Curitiba, 15 (4): 977-983.

Lawton, J.H.; M. MacGarvin \& P.A. Heads. 1987. Effects of altitude on the abundance and species richness of insect herbivores on bracken. Journal of Animal Ecology, Oxford, 56: 147-160.

Liewehr, J. \& W.S. Cranshaw. 1991. Alate aphid trap capture over different background colors and different background patterns. Southwestern Entomologist, Dallas, 16: 13-18.

Mackenzie, A.; A.F.G. Dixon \& P. Kindlmann. 1994. The relationship between the regional number of aphid species and plant species diversity. European Journal Entomology, Ceske Budejovice, 91: 135-138.

Martin, J.H. 1983. The identification of common aphid pests of tropical agriculture. Tropical Pest Management, Brisbane, 29: 395-411.

Moreira, C. 1925. Pulgão do Brasil. Rio de Janeiro, Instituto Biológico de Defesa Agrícola, 34p.

Odum, E.P. 1988. Ecologia. Rio de Janeiro, Editora Guanabara, $434 \mathrm{p}$.

Randall, M.G.M. 1982. The dynamics of an insect population throughout its altitudinal distributios: Coleophora alticolella (Lepidoptera) in Northern England. Journal of Animal Ecology, Oxford, 51: 993-1016.

Ribeiro, S.P.; M.A.A. Carneiro \& G.W. Fernandes. 1994. Distribution of Brachypnoea (Coleoptera: Chrysomelidae) in an altitudinal gradient in a Brazilian savanna vegetation. Phytophaga, Palermo, 6: 29-33.

RoBERT, Y. 1987. Aphids and their environment - dispersion and migration, p. 299-310. In: A.K. Minks \& P. Harrewijn. Aphids their biology, natural enemies and control. Amsterdam, Elsevier Science Publishers, vol. 2A, 450p.

Silva, A.G.A.; C.R. Gonçalves; D.M. Galvão; A.J.L. Gançalves; J. Gomes; M.N. Silva \& L. Simoni. 1968. Quarto catálogo dos insetos que vivem nas plantas do Brasil, seus parasitas e predadores. Rio de Janeiro, Ministério da Agricultura, parte 2 , tomo $1,622 \mathrm{p}$.

Sousa-Silva, C.R. \& F.A. Ilharco. 1995. Afídeos do Brasil e suas plantas hospedeiras (lista preliminar). São Carlos, EDUFSCar, 85p.

Trejo-Loyo, A.G.; Peña-Martinez, R. \& A. Marin-Jarillo. 2004. Notes on the biology and ecology of Aphis spiraecola Patch in Northern Morelos, Mexico, p. 87-91. In: J.C. Simon; C.A. 
Dedryver; C. Rispe \& M. Hullé (Eds). Aphids in a new millennium. Paris, INRA, 549p.

TuRnER, B.D. \& E. BROADHEAD. 1974. The diversity and distribution of psocid populations on Mangifera indica L. in Jamaica and their relationship to altitude and micro-epiphyte diversity. Journal of Animal Ecology, British Ecological Society, Oxford, 43: 173-190.

Walters, K.F.A. \& A.F.G. Dixon. 1984. The effect of temperature and wind on the flight activity of cereal aphids. Annals of Applied Biology, London, 104: 17-26.

Wolda, H. 1978. Seasonal fluctuations in rainfall, food and abundance of tropical insects. Journal of Animal Ecology, Oxford, 47: 369-381.

WolDA, H. 1987. Altitude, habitat and tropical insect diversity. Biological Journal of the Linnean Society, London, 30: 313-323.

Received in 17.II.2005; accepted in 30.IX.2005. 